
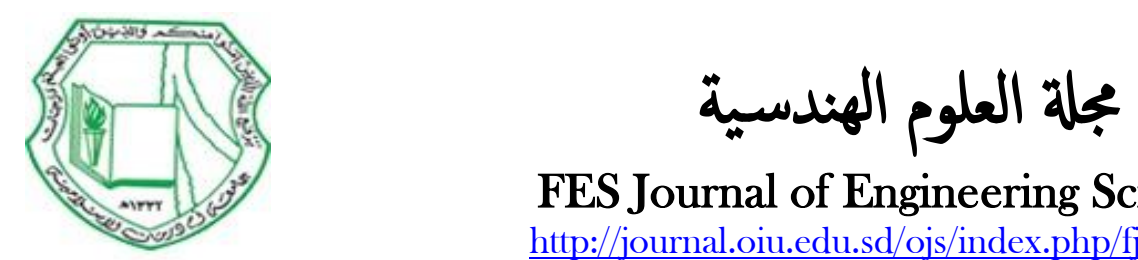

FES Journal of Engineering Sciences

http://journal.oiu.edu.sd/ojs/index.php/fjes/index

\title{
Finite Element Analysis of Rectangular Beams
}

\author{
Abusamra A. Attaelmanan ${ }^{1,2}$ and Abdelhameed H. Mohammed Ali ${ }^{1, *}$ \\ 1 Civil Engineering Department, Omdurman Islamic University, Omdurman, Sudan. \\ 2 Civil Engineering Dept. Faculty of Engineering, Al Baha University, Saudi Arabia. \\ * hameedsarwor@gmail.com \\ Article history: Received 12 February 2019, Received in revised form 05 March 2019, Accepted 06 March 2019
}

\begin{abstract}
This paper is concerned with the analysis of simply supported beam using MATLAB programming language and structural analysis program SAP2000. The beam was discretized into rectangular elements using finite element method. Three patterns of different dimensions and numbers of rectangular elements were used to verify the results of vertical displacements and stresses obtained by MATLAB and SAP 2000.The development of four noded isoparametric quadrilateral membrane elements in MATLAB programming language is presented. The membrane elements developed are plane strain condition. The considered patterns were analyzed as shell elements using SAP2000. A finite element program is also developed using MATLAB to check the accuracy of the developed elements.
\end{abstract}

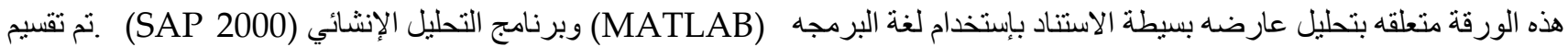

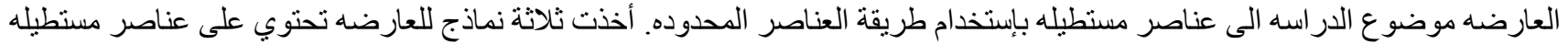

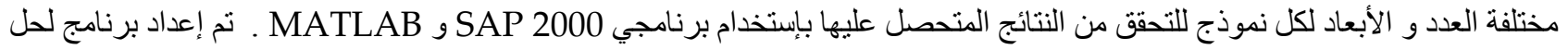

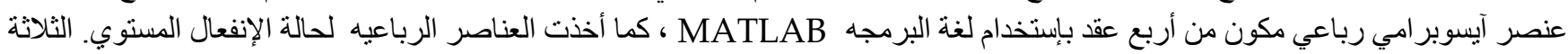

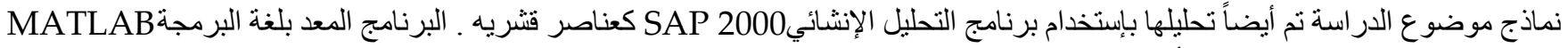

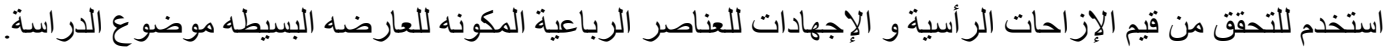

Keywords: MATLAB , beam ,elements, finite, stresses, noded, isoparametric.

\section{INTRODUCTION}

The finite element method has become an important tool in structural analysis. It became popular with the development of digital computers since they allow engineers to solve large systems of equations quickly and efficiently. In most structural analysis applications it is necessary to compute vertical displacements and stresses at various points of interest. In the finite element method, the finite element model is created by dividing the structure into a number of finite elements. Each element is interconnected by nodes. The selection of elements for modeling the structure depends upon the behavior and geometry of the structure being analyzed. The modeling pattern, which is generally called mesh for the finite element method, is a very important part of the modeling process.

The results obtained from the analysis depend upon the selection of the finite elements and the mesh size. Although the finite element model does not behave exactly like the actual structure, it is possible to obtain sufficiently accurate results for most practical applications.

\section{MATERIAL AND METHODS}

The formulation of the element stiffness matrix for the four noded quadrilateral plane stress elements is described below. To develop the isoparametric quadrilateral plane stress element, the parent element must be defined in the natural coordinate system $(\mathrm{r}, \mathrm{s})$ [1].

The relationship between the natural coordinate system and the global coordinate 
system can be defined using Lagrange interpolating functions.

$$
X_{(r, s)}=\sum_{i=1}^{4}\left(N_{i} x_{i}\right)
$$

and

$$
Y_{(r, s)}=\sum_{i=1}^{4}\left(N_{i} y_{i}\right)
$$

Similarly, the relationship between displacements in the natural coordinate system and the nodal displacements can be written in the following manner,

$$
\begin{gathered}
\mathcal{u}(r, s)=\sum_{i=1}^{4}\left(N_{i} u_{i}\right) \\
\mathcal{v}(r, s)=\sum_{i=1}^{4}\left(N_{i} v_{i}\right)
\end{gathered}
$$

where $N_{1}, N_{2}, N_{3}, N_{4}$ are the shape functions for the four noded quadrilateral elements in the natural coordinate system. The shape functions are,

$$
\begin{aligned}
& N_{1}=\frac{1}{4}(1-r)(1-s) ; \quad N_{2}=\frac{1}{4}(1+r)(1-s) ; \\
& N_{3}=\frac{1}{4}(1+r)(1+s) ; \quad N_{4}=\frac{1}{4}(1-r)(1+s)
\end{aligned}
$$

The Jacobian matrix can be defined as,

$$
[J]=\left[\begin{array}{ll}
J_{11} & J_{12} \\
J_{21} & J_{22}
\end{array}\right]
$$

The determinant of the Jacobian is,

$$
|J|=J_{11} J_{22}-J_{12} J_{21}
$$

and the inverse of the Jacobian matrix is,

$$
[J]^{-1}=\frac{1}{|J|}\left[\begin{array}{cc}
J^{*}{ }_{22} & -J^{*}{ }_{12} \\
-J^{*}{ }_{21} & J^{*}{ }_{11}
\end{array}\right]
$$

From Equations (5) the terms in the Jacobian matrix can be obtained as,

$$
\begin{aligned}
& J_{11}=\frac{\partial x}{\partial r}=\sum_{i=1}^{4} \frac{\partial N_{i}}{\partial r} x_{i} ; J_{12}=\frac{\partial y}{\partial r}=\sum_{i=1}^{4} \frac{\partial N_{i}}{\partial r} y_{i} ; \\
& J_{21}=\frac{\partial x}{\partial s}=\sum_{i=1}^{4} \frac{\partial N_{i}}{\partial s} x_{i} ; J_{22}=\frac{\partial y}{\partial s}=\sum_{i=1}^{4} \frac{\partial N_{i}}{\partial s} y_{i}
\end{aligned}
$$

Thus, the strain-displacement relationships for the four node isoperimetric quadrilateral element are,

$$
\left\{\begin{array}{c}
\varepsilon_{x} \\
\varepsilon_{y} \\
\gamma_{x y}
\end{array}\right\}=\left\{\begin{array}{c}
\frac{\partial u}{\partial x} \\
\frac{\partial v}{\partial y} \\
\frac{\partial u}{\partial y}+\frac{\partial v}{\partial x}
\end{array}\right\}
$$

The derivatives of the horizontal displacement with respect to $\mathrm{x}$ and $\mathrm{y}$ in terms of Jacobian matrix are, $\left\{\begin{array}{l}\frac{\partial u}{\partial x} \\ \frac{\partial u}{\partial y}\end{array}\right\}=\frac{1}{|J|}\left[\begin{array}{cc}J^{*}{ }_{22} & -J^{*}{ }_{12} \\ -J^{*}{ }_{21} & J^{*}{ }_{11}\end{array}\right]\left\{\begin{array}{l}\frac{\partial u}{\partial r} \\ \frac{\partial u}{\partial s}\end{array}\right\}$

Similarly, the derivatives of the vertical displacement with respect to $\mathrm{x}$ and $\mathrm{y}$ are,

$$
\left\{\begin{array}{l}
\frac{\partial v}{\partial x} \\
\frac{\partial v}{\partial y}
\end{array}\right\}=\frac{1}{|J|}\left[\begin{array}{cc}
J^{*}{ }_{22} & -J^{*}{ }_{12} \\
-J^{*}{ }_{21} & J^{*}{ }_{11}
\end{array}\right]\left\{\begin{array}{l}
\frac{\partial v}{\partial r} \\
\frac{\partial v}{\partial s}
\end{array}\right\}
$$

From Equations (9), (10), and (11), the strain-displacement relationships can be obtained in terms of natural co-ordinates $\mathbf{r}$ and $\mathbf{s}$ as,

$$
\left\{\begin{array}{l}
\varepsilon_{x} \\
\varepsilon_{y} \\
\gamma_{x y}
\end{array}\right\}=[A]\left\{\begin{array}{l}
\frac{\partial u}{\partial r} \\
\frac{\partial u}{\partial s} \\
\frac{\partial v}{\partial r} \\
\frac{\partial v}{\partial s}
\end{array}\right\}
$$

where,

$$
[A]=\frac{1}{|J|}\left[\begin{array}{cccc}
J_{22} & J_{12} & 0 & 0 \\
0 & 0 & -J_{21} & J_{11} \\
-J_{21} & J_{11} & J_{22} & -J_{12}
\end{array}\right]
$$

Now, from Equations (3) and (4) the following relationships are obtained,

$$
\frac{\partial u}{\partial r}=\sum_{i=1}^{4} \frac{\partial N_{i}}{\partial r} u_{i}
$$

$$
\begin{aligned}
& \frac{\partial u}{\partial s}=\sum_{i=1}^{4} \frac{\partial N_{i}}{\partial s} u_{i} \\
& \frac{\partial v}{\partial r}=\sum_{i=1}^{4} \frac{\partial N_{i}}{\partial r} v_{i} \\
& \frac{\partial v}{\partial r}=\sum_{i=1}^{4} \frac{\partial N_{i}}{\partial r} v_{i}
\end{aligned}
$$

Equation (14), can be rewritten in matrix form as,

$$
\left\{\begin{array}{l}
\frac{\partial u}{\partial r} \\
\frac{\partial u}{\partial s} \\
\frac{\partial v}{\partial r} \\
\frac{\partial v}{\partial s}
\end{array}\right\}=\left[\begin{array}{cccccccc}
\frac{\partial N_{1}}{\partial r} & 0 & \frac{\partial N_{2}}{\partial r} & 0 & \frac{\partial N_{3}}{\partial r} & 0 & \frac{\partial N_{4}}{\partial r} & 0 \\
\frac{\partial N_{1}}{\partial s} & 0 & \frac{\partial N_{2}}{\partial r} & 0 & \frac{\partial N_{3}}{\partial r} & 0 & \frac{\partial N_{4}}{\partial r} & 0 \\
0 & \frac{\partial N_{1}}{\partial r} & 0 & \frac{\partial N_{2}}{\partial r} & 0 & \frac{\partial N_{3}}{\partial r} & 0 & \frac{\partial N_{4}}{\partial r} \\
0 & \frac{\partial N_{1}}{\partial r} & 0 & \frac{\partial N_{2}}{\partial r} & 0 & \frac{\partial N_{3}}{\partial r} & 0 & \frac{\partial N_{4}}{\partial r}
\end{array}\right]\left\{\begin{array}{l}
u_{1} \\
v_{1} \\
u_{2} \\
v_{2} \\
u_{3} \\
v_{3} \\
u_{4} \\
v_{4}
\end{array}\right\}
$$

Now, using Equations (10) to (13) the following relationships are obtained,

$$
\{\varepsilon\}=[A \rrbracket G]\{U\}
$$


where,

$$
[G]=\left[\begin{array}{cccccccc}
\frac{\partial N_{1}}{\partial r} & 0 & \frac{\partial N_{2}}{\partial r} & 0 & \frac{\partial N_{3}}{\partial r} & 0 & \frac{\partial N_{4}}{\partial r} & 0 \\
\frac{\partial N_{1}}{\partial s} & 0 & \frac{\partial N_{2}}{\partial r} & 0 & \frac{\partial N_{3}}{\partial r} & 0 & \frac{\partial N_{4}}{\partial r} & 0 \\
0 & \frac{\partial N_{1}}{\partial r} & 0 & \frac{\partial N_{2}}{\partial r} & 0 & \frac{\partial N_{3}}{\partial r} & 0 & \frac{\partial N_{4}}{\partial r} \\
0 & \frac{\partial N_{1}}{\partial r} & 0 & \frac{\partial N_{2}}{\partial r} & 0 & \frac{\partial N_{3}}{\partial r} & 0 & \frac{\partial N_{4}}{\partial r}
\end{array}\right]
$$

Thus,

$$
\{\varepsilon\}=[B]\{U\}
$$

where,

$$
\begin{array}{r}
{[B]=[A \rrbracket[G]} \\
\text { Equation }
\end{array}
$$

represents isoparametric quadrilateral elements. The element stiffness matrix of the four noded isoparametric quadrilateral elements for the plane stress condition is given by;

$$
[k]=\iint_{A}[B]^{T}[D][B] d A
$$

Since the strain displacement matrix is in terms of the natural coordinates, therefore Equation (20) must be integrated with respect to the natural coordinates.

Substituting for $d A$,

$$
d A=d x d y=|J| d r d s
$$

Therefore, Equation (20) can be rewritten as,

$$
[k]_{8 * 8}=h \int_{-1}^{1} \int_{-1}^{1}\left[B_{(r, s)}\right]^{T}[D]\left[B_{(r, s)}\right] J_{(r, s)} d r d s
$$

where, $\mathrm{h}=$ thickness of the element.

The element stiffness matrix can be obtained using $(2 * 2)$ Gauss quadrature,

$$
[k]_{8 * 8}=h \sum_{j=1}^{2} \sum_{i=1}^{2} w_{j} w_{i}\left[B\left(r_{i}, s_{j}\right)\right]^{T}[D]\left[B\left(r_{i}, s_{j}\right] J\left(r_{i}, s_{j}\right) \mid d r d s\right.
$$

"Refs. [2]-[4]".

The six MATLAB functions used for the bilinear quadrilateral element are:

1. Bilinear Quad Element Area( $x_{1}, y_{1}, x_{2}, y_{2}, x_{3}, y_{3}$, $\left.\mathbf{x}_{4}, \mathbf{y}_{4}\right)$ - This function returns the element area given the coordinates of the first node $\left(\mathrm{x}_{1}, \mathrm{y}_{1}\right)$, the coordinates of the second node $\left(x_{2}, y_{2}\right)$, the coordinates of the third node $\left(\mathrm{x}_{3}, \mathrm{y}_{3}\right)$, and the coordinates of the fourth node $\left(\mathrm{x}_{4}, \mathrm{y}_{4}\right)$.

2. Bilinear Quad Element Stiffness $\left(E, v, h, x_{1}, y_{1}\right.$, $\left.\mathbf{x}_{2}, \mathbf{y}_{2}, \mathbf{x}_{3}, \mathbf{y}_{3}, \mathbf{x}_{4}, \mathbf{y}_{4}, \mathbf{p}\right)$ - This function calculates the element stiffness matrix for each bilinear quadrilateral element with modulus of elasticity E, Poisson's ratio $v$, thickness $h$, and coordinates $\left(\mathrm{x}_{1}, \mathrm{y}_{1}\right)$ for the first node, $\left(\mathrm{x}_{2}, \mathrm{y}_{2}\right)$ for the second node, $\left(\mathrm{x}_{3}, \mathrm{y}_{3}\right)$ for the third node, and $\quad\left(\mathrm{x}_{4}, \mathrm{y}_{4}\right)$ for the fourth node. Use $p=1$ for cases of plane stress and $\mathrm{p}=2$ for cases of plane strain. It returns the 8 $\times 8$ element stiffness matrix $\mathrm{k}$.

3. BilinearQuadElementStiffness $2\left(E, v, h, x_{1}, y_{1}\right.$, $\left.\mathbf{x}_{2}, \mathbf{y}_{2}, \mathbf{x}_{3}, \mathbf{y}_{3}, \mathbf{x}_{4}, \mathbf{y}_{4}, \mathbf{p}\right)$ - This function calculates the element stiffness matrix for each bilinear quadrilateral element with modulus of elasticity E, Poisson's ratio $v$, thickness $h$, and coordinates $\left(x_{1}, y_{1}\right)$ for the first node, $\left(x_{2}, y_{2}\right)$ for the second node, $\left(x_{3}, y_{3}\right)$ for the third node, and $\left(x_{4}, y_{4}\right)$ for the fourth node. Use $\mathrm{p}=1$ for cases of plane stress and $p=2$ for cases of plane strain. It returns the $8 \times 8$ element stiffness matrix $\mathrm{k}$. This function uses a different form of the element equations but produces exactly the same result as the function Bilinear Quad Element Stiffness.

4. Bilinear Quad Assemble (K, k, i, j, $\mathbf{m}, \mathbf{n})$ - This function assembles the element stiffness matrix $\mathrm{k}$ of the bilinear quadrilateral element joining nodes $i, j, m$, and $n$ into the global stiffness matrix $K$. It returns the $2 n \times 2 n$ global stiffness matrix $K$ every time an element is assembled.

5. Bilinear Quad Element Stresses( $\mathrm{E}, \boldsymbol{v}, \mathrm{x}_{1}, \mathrm{y}_{1}, \mathrm{x}_{2}$, $\left.\mathbf{y}_{2}, \mathbf{x}_{3}, \mathbf{y}_{3}, \mathbf{x}_{4}, \mathbf{y}_{4}, \mathbf{p}, \mathbf{u}\right)$ This function calculates the element stresses using the modulus of elasticity $\mathrm{E}$, Poisson's ratio $v$, the coordinates $\left(\mathrm{x}_{1}, \mathrm{y}_{1}\right)$ for the first node, $\left(x_{2}, y_{2}\right)$ for the second node, $\left(x_{3}, y_{3}\right)$ for the third node, $\left(\mathrm{x}_{4}, \mathrm{y}_{4}\right)$ for the fourth node, and the element displacement vector $u$. Use $p=1$ for cases of plane stress and $p=2$ for cases of plane strain. It returns the stress vector for the element.

6. Bilinear Quad Element P Stresses (sigma) This function calculates the element principal stresses using the element stress vector sigma. It returns a $3 \times 1$ vector in the form [sigma1 sigma2 theta $]^{\mathrm{T}}$ where sigma 1 and sigma 2 are the principal stresses for the element and theta is the principal angle [3].

The following is a listing of the MATLAB source code for each function; 


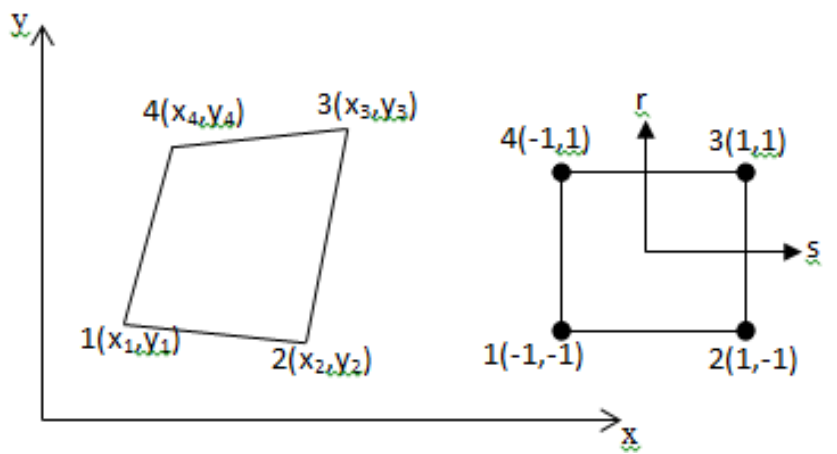

(a) Global Co-ordinates (b) Natural Co-ordinates Fig.1. Typical Bilinear Quadrilateral Element.

\section{RESULTS AND DISCUSSION}

All patterns are simply supported beam of length $6.0 \mathrm{~m}$ and have cross-sectional area of $\left(0.3 \mathrm{~m}^{*} 0.5 \mathrm{~m}\right)$. The concrete modulus of elasticity and poison's ratio are $26^{*} 10^{6} \mathrm{kN} / \mathrm{m}^{2}$ and 0.2 respectively. Three patterns were discredited into 20, 24 and 30 rectangular elements. The load applied to those patterns is $100 \mathrm{kN} / \mathrm{m}$, which were transferred into point loads on the top joints of beam. For the three beams patterns, the point loads of $60 \mathrm{kN}, 50 \mathrm{kN}$ and $40 \mathrm{kN}$ were applied at interior joints and at exterior joints were $30 \mathrm{kN}$, $25 \mathrm{kN}$ and $20 \mathrm{kN}$ respectively. The dimensions of rectangular element for each pattern are given on Fig. $2-4$.

Firstly, analysis of three patterns is carried using MATLAB, It is assumed that all discretized rectangular elements are two dimensional plane strain bilinear quadrilateral beam analyzed using MATLAB programming language and structural analysis program SAP 2000 has been carried out. Since the basic reference of comparison is the MATLAB. The general comparison of results for the three patterns of simply supported reinforced concrete Figs. 7 - 15 show the span - vertical deflection and span - stress diagrams for all beam's patterns. Pattern (1) as shown in Fig.5. It has been assumed that all discretized elements are flat shell elements.

The linear static analysis of beams patterns may be confirmed by studying the distribution of deflections and stresses over the span of beam. Secondly, the results of analysis using structural analysis SAP 2000 was presented in stresses distribution and deflections for all beams.

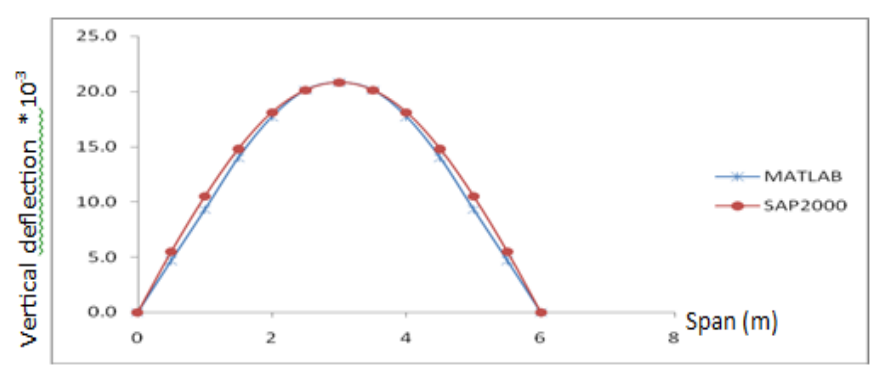

Fig.8. Span-vertical deflection diagram of beam pattern (1).

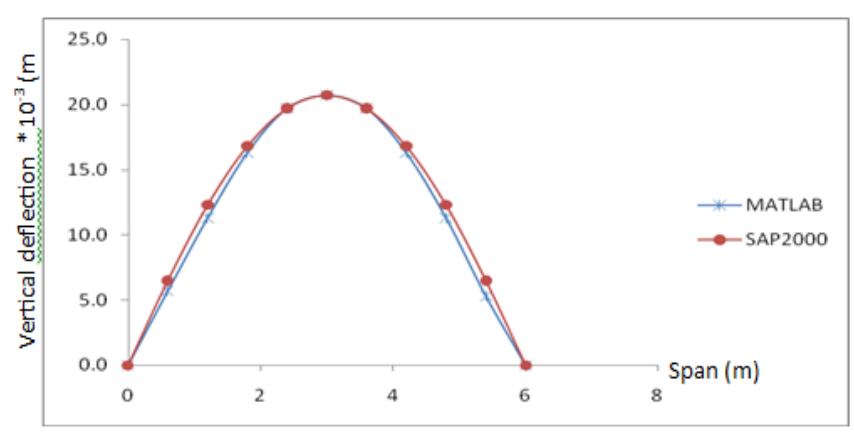

Fig.9. Span - vertical deflection diagram of beam pattern (2).

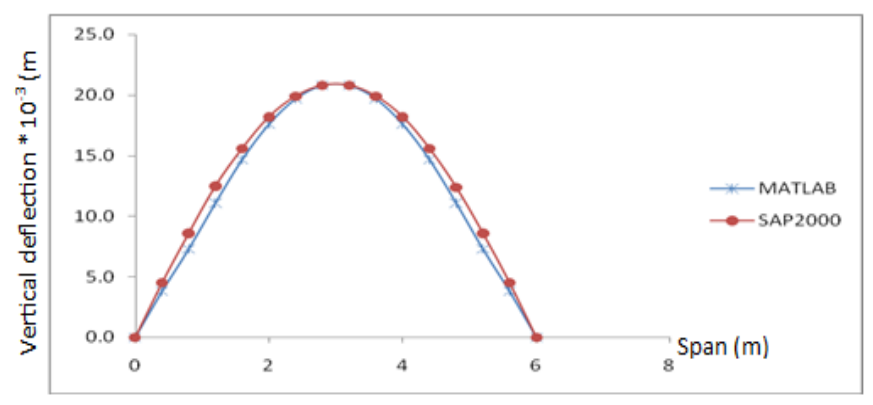

Fig.10. Span - vertical deflection diagram of beam pattern (3)

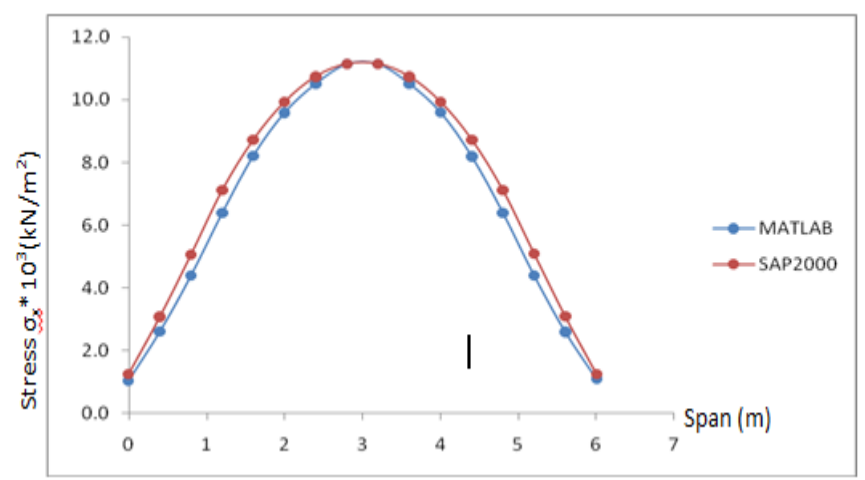

Fig.11. Span - stress $\sigma x$ diagram of beam pattern (1) 


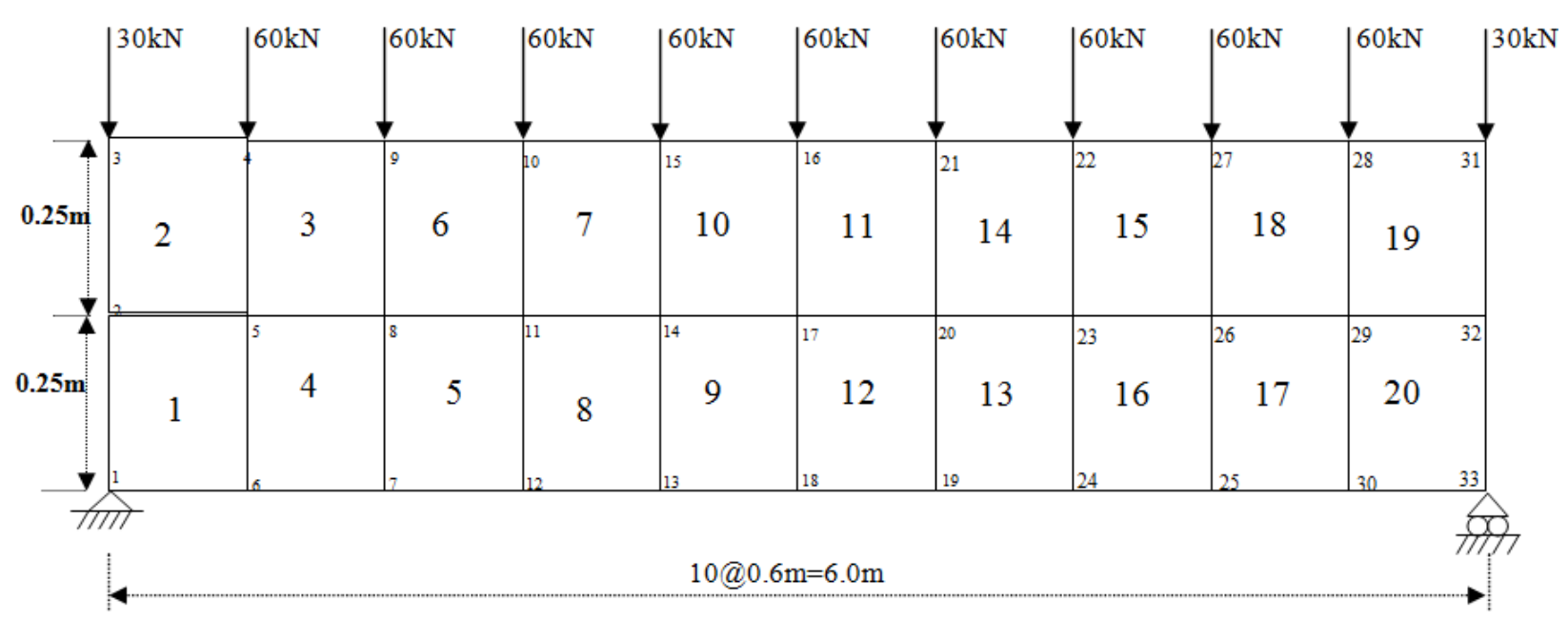

Fig.2. Beam pattern (1)

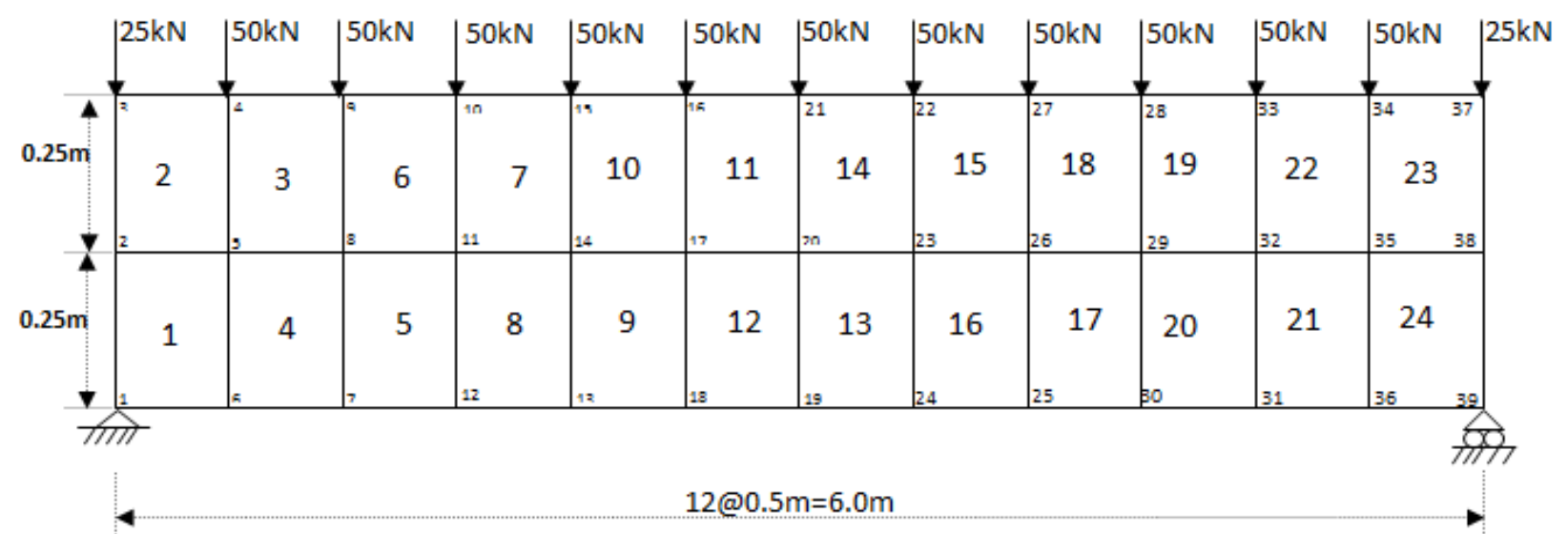

Fig.3. Beam pattern (2)

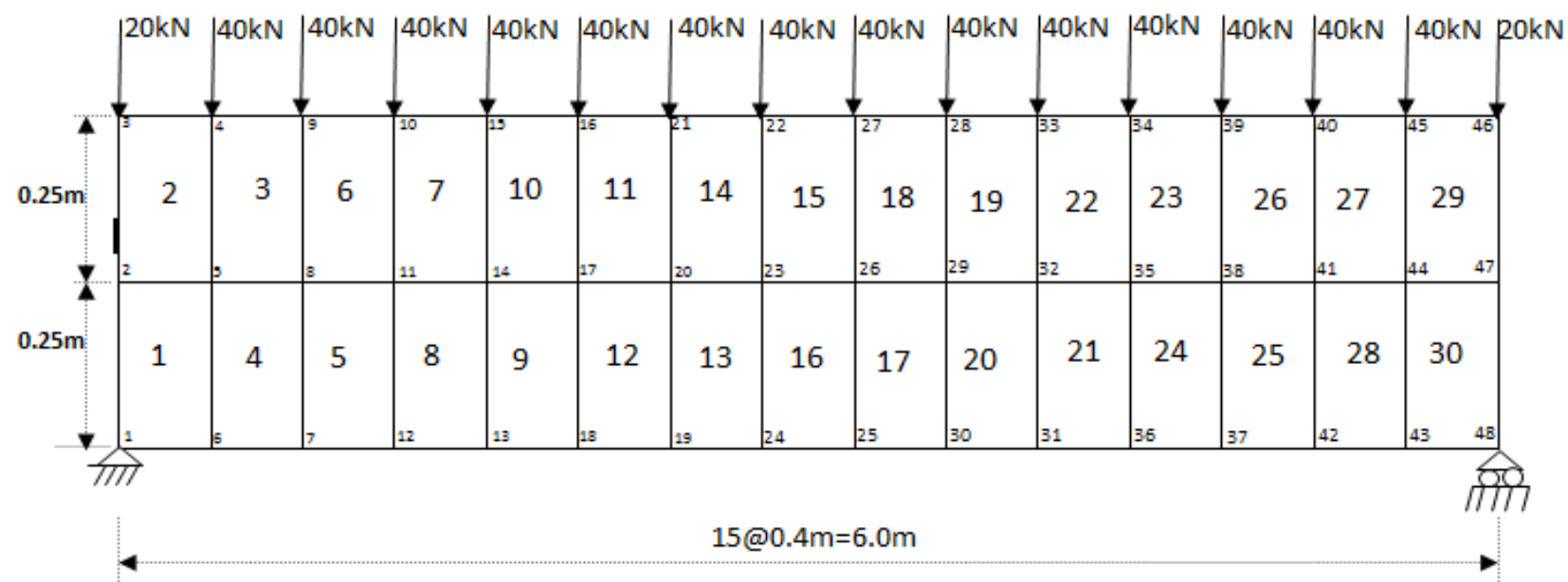

Fig.4. Beam pattern (3) 


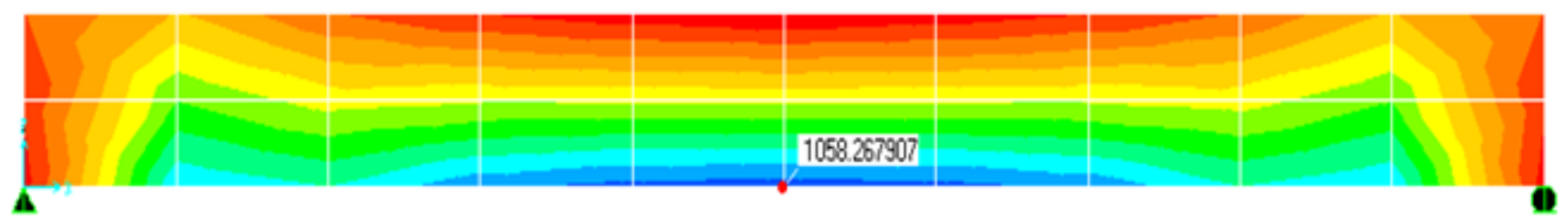

\begin{tabular}{|c|c|c|c|c|c|c|c|c|c|c|c|c|c|}
\hline-1.33 & -1.14 & $-0,95$ & -0.76 & -0.57 & -0.38 & -0.19 & 0.00 & 0.19 & 0.38 & 0.57 & 0.76 & 0.95 & 1.1 \\
\hline & & & & & & & & & & & $\sqrt{65}$ & $\overline{\text { GLOBAL }}$ & $\begin{array}{l}\overline{K N}, \mathrm{~m}, \mathrm{C} \\
\end{array}$ \\
\hline
\end{tabular}

Fig.5. Stress ( $\sigma \mathrm{x})$ contour of beam pattern (1)

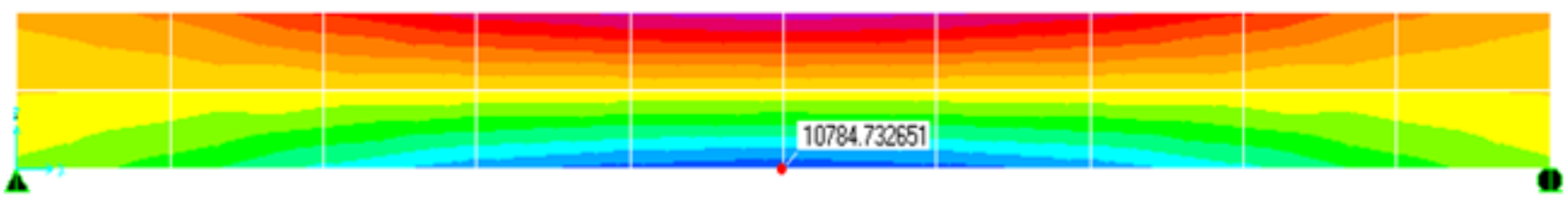

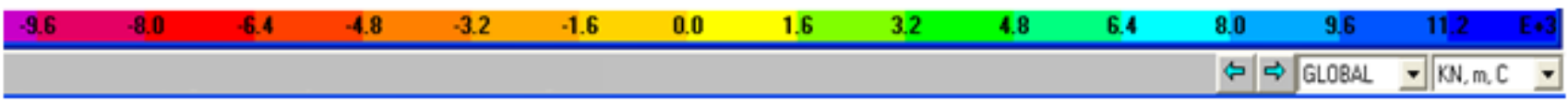

Fig.6. Stress ( $\sigma y)$ contour of beam pattern (1)
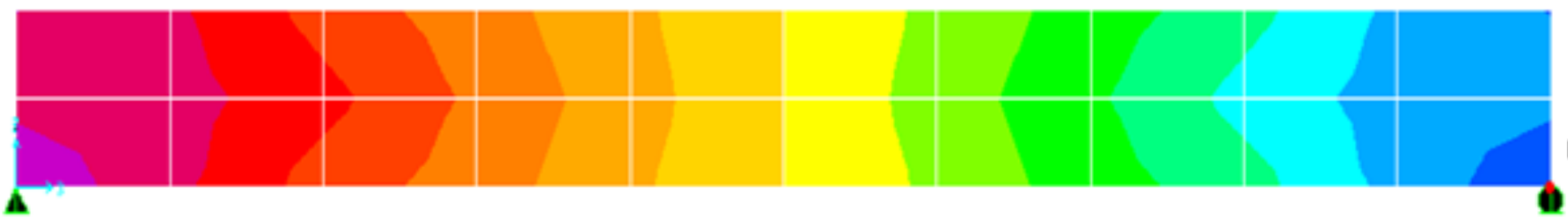

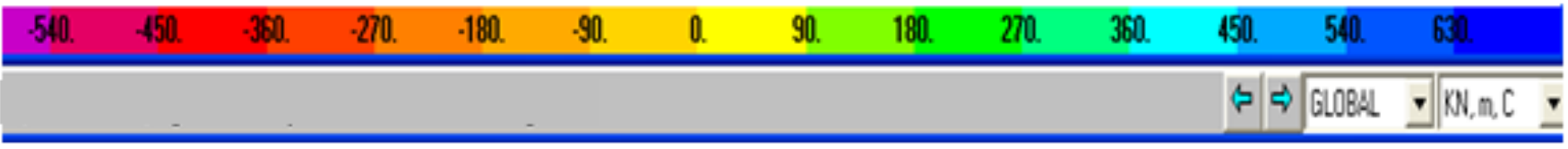

Fig.7. Stress $\left(\tau_{\mathrm{xy}}\right)$ contour of beam pattern (1)

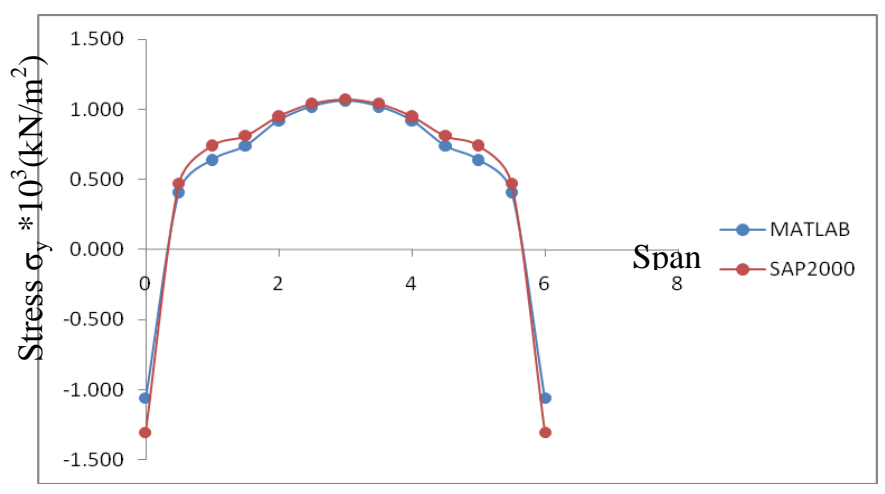

Fig.12. Span - stress oy diagram of beam pattern (1)

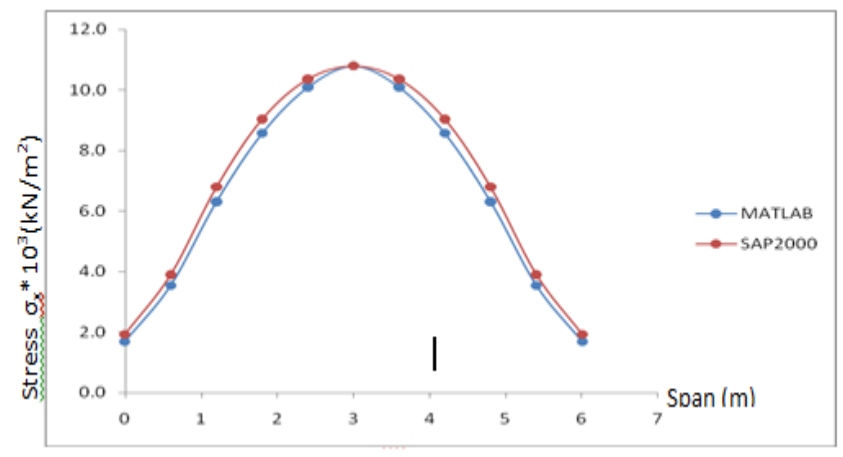

Fig.13. Span - stress $\sigma_{x}$ diagram of beam pattern (2) 


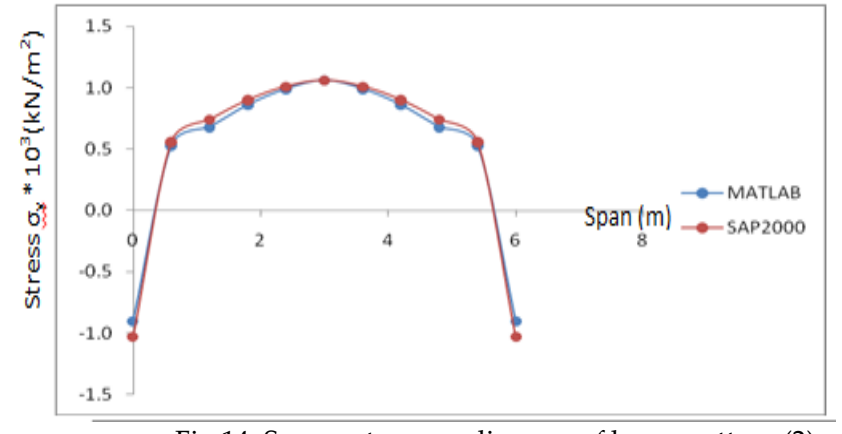

Fig.14. Span - stress oy diagram of beam pattern (2)

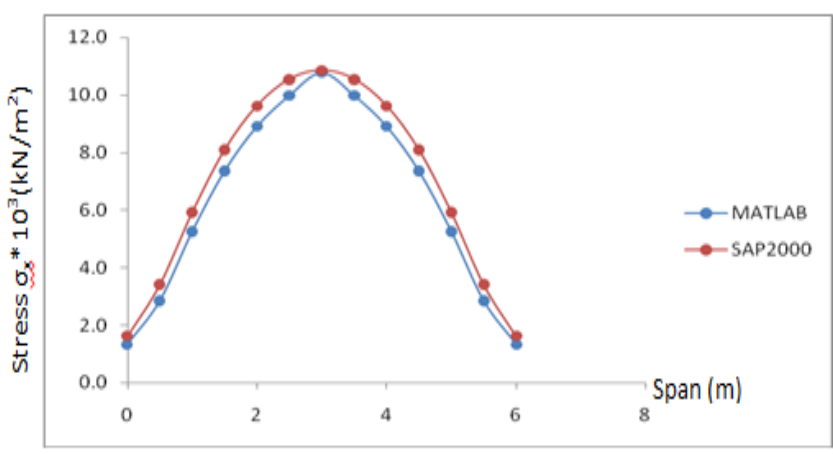

Fig.15. Span - stress $\sigma x$ diagram of beam pattern (3)

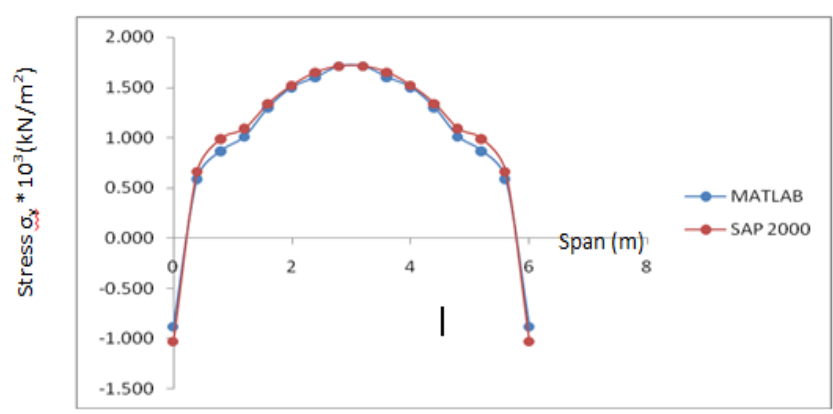

Fig.16. Span - stress oy diagram of beam pattern (3)

\section{CONCLUSIONS}

The analysis of three patterns using MATLAB program was compared with those obtained from SAP2000 in order to verify the accuracy of the developed program. All patterns have shown increments in deflections and stress distributions in comparison with MATLAB. The comparison can be summarized as follows:

1-In the case of pattern (1vertical displacements and stresses have increase $(0-14 \%)$ and $(0-12.4 \%)$ respectively.

2-In the case of pattern (2), the vertical displacements have increased by about (1- 17\%), and stresses have increased by zero to $19 \%$.
3-Finally, in the case of pattern (3), the vertical displacements have increased about (0 - $18.4 \%$ ) and stresses have increased by zero to $15.3 \%$.

An important result was made when analyzing three patterns using plane strain four noded rectangular elements using MATLAB programming language and structural analysis program SAP2000. It was found that the nodes near supports gave quite large differences about $19 \%$ in vertical displacements and stress distribution. The central nodes gave differences in displacements and stresses less that $1 \%$ for all patterns.

\section{References}

[1] Cook R. D., Malkus D. S., and Plesha M. E., Concepts and Applications of Finite Element Analysis, 3rd ed., John Wiley \& Sons, 1989.

[2] McNeal R. H., A Simple Quadrilateral Shell Element, Computers and Structures, Vol. 8, 1978, pp. 175-183.

[3] Peter I. Kattan- MATLAB Guide to Finite Elements An Interactive Approach, Springer Berlin Heidelberg, New York, Springer-Verlag Berlin Heidelberg 2008.

[4] Bathe K. J., Wilson E. L., Numerical Methods in Finite Element Analysis, Prentice-Hall Inc., 1976.

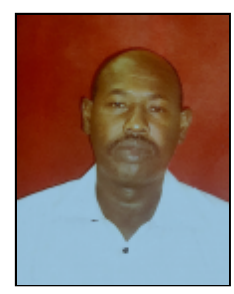

Abusamra Awad Attaelmanan received,his bachelor of science degree in Civil engineering from Omdurman Islamic University, Sudan in 1996 ; the master of science in Civil engineering (Structures) from Sudan University of Science and Technology, Khartoum, Sudan, 2000 and Ph.D of science in Civil engineering (Structures) from Rostov State University of Civil Engineering, Russia, 2008. He has been appointed as a lecturer at Faculty of Engineering, Al Baha Universit Al Baha, Saudi Arabia.

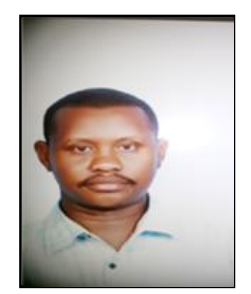

Abdelhameed Hamed Mohammed Ali received his bachelor of science degree in Civil engineering from Omdurman Islamic University, Sudan in 2005; the master of science in Civil engineering (Structures) from Karary University, Sudan in 2012 and Ph.D of science in Civil engineering (Structures) from Karary University , Sudan in 2016. He has been appointed as a lecturer at Omdurman Islamic University, Khartoum, Sudan since 2006. 